\title{
Ginkgo suppresses atherosclerosis through downregulating the expression of connexin 43 in rabbits
}

\author{
Jian Ming Wei ${ }^{1}$, Xin Wang ${ }^{1}$, Hui Gong ${ }^{1}$, Yi Jun Shi' ${ }^{1}$ Yunzeng Zou ${ }^{2}$
}

1Department of Cardiology, Jinshan Hospital, Fudan University, Shanghai, China 2Shanghai Institute of Cardiovascular Diseases, Zhongshan Hospital and Institutes of Biomedical Sciences, Fudan University, Shanghai, China

Submitted: 14 July 2012

Accepted: 9 November 2012

Arch Med Sci 2013; 9, 2: 340-346

DOI: $10.5114 /$ aoms.2013.34416

Copyright @ 2013 Termedia \& Banach

\section{Abstract}

Introduction: Ginkgo biloba extract (GBE) EGb761 is widely used for cardiovascular prevention. Here, we investigated the effects of GBE on atherosclerotic lesion development in rabbits with a high-fat diet.

Material and methods: Forty New Zealand white male rabbits were randomly divided into four groups. The first two were the normal diet group (C) and the high-fat group (HF). The remaining two groups were those who received a high cholesterol diet supplemented with either the standard drug (simvastatin $2 \mathrm{mg} / \mathrm{kg} /$ day) or GBE (3 mg/kg/day). At 12 weeks, histopathological and chemical analyses were performed.

Results: Plasma lipid measurement showed that GBE inhibited high-fat dietinduced increase of serum triglyceride (TG), total cholesterol (TC), and low density lipoprotein cholesterol (LDL-C) by $59.1 \%(0.9 \pm 0.24 \mathrm{mmol} / / \mathrm{lvs} .2 .2$ $\pm 0.4 \mathrm{mmol} / \mathrm{l}), 18.2 \%(31.1 \pm 1.4 \mathrm{mmol} / \mathrm{l}$ vs. $38.0 \pm 0.4 \mathrm{mmol} / \mathrm{l})$ and $15 \%(28.9$ $\pm 1.3 \mathrm{mmol} / / \mathrm{l}$ vs. $34.0 \pm 1.0 \mathrm{mmol} / \mathrm{l})$, respectively, at 12 weeks $(p<0.01)$. The en face Sudan IV-positive lesion area of the aorta in the GBE group $(51.7 \pm 3.1 \%)$ was significantly lower compared with that in the HF group $(88.2 \pm 2.2 \%$; $p<0.01$ ). The mean atherosclerotic lesion area of the GBE group was reduced by $53.2 \%$ compared with the HF group $(p<0.01)$. Immunohistochemistry and western blot analysis showed that GBE markedly suppressed high-fat dietinduced upregulation of connexin $43(C \times 43)$ in rabbits $(p<0.01)$.

Conclusions: Thus, our study revealed that GBE prevented atherosclerosis progress through modulating plasma lipid, suppressing atherosclerotic lesion development, and attenuating the expression of Cx43 protein.

Key words: atherosclerosis, Ginkgo biloba extract, connexin 43, plasma lipid, highfat diet.

\section{Introduction}

Atherogenesis is a multi-step process, which includes the accumulation of oxidized low density lipoprotein or low-density lipoprotein (LDL) within the blood vessel wall and an ensuing inflammatory cascade, leading to later stages of plaque development and thrombosis that are difficult to reverse [1]. Moreover, atherosclerosis is a chronic disease, and undetected in the early stage [1]. Thus, management of atherosclerosis, especially at the early stage, proves difficult. Even though treatments for clinical

\author{
Corresponding author: \\ Jian Ming Wei MD, PhD \\ Department of Cardiology \\ Jinshan Hospital \\ Fudan University \\ Shanghai 200540, China \\ Phone: +86 21341899 \\ 90-5343 \\ Fax: +862167226910 \\ E-mail: jianming.wei \\ @yahoo.cn
}


manifestations of atherosclerosis are available, they tend to be plagued by several inherent drawbacks. Many pharmaceutical candidates and theoretically cardioprotective drugs fall short in a clinical setting, because of their off-target effects and low efficacy at tolerated doses [2-4]. Statins, one of the cholesterol lowering drugs, are proven to decrease the mortality of coronary heart disease. However, statins fail to rescue from acute ischemic events and prevent cellular uptake of oxidatively modified LDL [3-6]. Anti-cytokine antibodies and cytokine secretion inhibitors can diminish inflammatory responses which deteriorate atherosclerosis [7, 8], but trials have not shown efficacy in reducing clinical endpoints $[9,10]$. Anti-chemokine therapy can reduce macrophage recruitment and block inflammation initiation. But these effects are limited by the interaction between chemokines and receptors [11]. Anti-platelet therapies prevent clot formation and aid clot breakup, but only address the latter stages of cardiovascular disease when clots are more likely to form $[12,13]$. Current status of drug therapy for atherosclerosis demonstrates the need for the development of alternative strategies and platforms [14, 15].

Ginkgo biloba extract (GBE), from Ginkgo biloba leaves, has been widely used as a therapeutic agent for cardiovascular and neurological disorders [16]. Clinical studies showed that GBE can reduce atherosclerotic nanoplaque formation and size in cardiovascular high-risk patients $[17,18]$. Experimental studies showed that GBE can inhibit nuclear factor $\kappa \mathrm{B}(\mathrm{NF}-\kappa \mathrm{B})$ activation induced by hydrogen peroxide $\left(\mathrm{H}_{2} \mathrm{O}_{2}\right)$ [19]. Ginkgo biloba extract can diminish cytokine-stimulated endothelial adhesiveness by downregulating intracellular reactive oxygen species formation, NF- $\mathrm{KB}$ and activator protein 1 (AP-1) activation, and adhesion molecule expression in human aortic endothelial cells (HAECs) [20]. It also inhibits production of pro-inflammatory cytokines interleukin $1 \beta$ (IL-1 $\beta$ ) and tumor necrosis factor $\alpha$ (TNF- $\alpha)$, but up-regulates the production of anti-inflammatory cytokines [21]. All of these studies support the notion that GBE may have potential implications in clinical atherosclerosis disease. GBE-mediated inhibition of ox-LDL-induced lipoperoxide levels in endothelial cells and patients appears to be an important mechanism by which GBE exhibits the atherosclerosis inhibiting effect [17, 18, 22]. Ginkgo biloba extract is also documented to downregulate vascular endothelial growth factor (VEGF) expression, inhibit VEGF-mediated permeability, and suppress monocyte/macrophage-derived foam cell formation by a novel heme oxygenase-1 (HO-1)-dependent regulation of cholesterol homeostasis in macrophages [23-26]. In the present study, we analyzed the effects of GBE on atherosclerosis in rabbits with a high-fat diet.

\section{Material and methods}

\section{Animal model}

Forty New Zealand white male rabbits (4 months old, weighing $2.2-2.5 \mathrm{~kg}$ ) were provided by the Laboratory Animal Centre of Shanghai Songlian (Shanghai, China) and individually caged in an air-conditioned room. There was an adaptation period of 7 days for animals before experiments. These rabbits were randomly divided into four groups: control group animals $(C ; n=10)$ were fed a regular diet for 12 weeks; atherosclerosis group animals (HF; $n=10)$ were fed a high fat diet supplemented with $1 \%(\mathrm{w} / \mathrm{w})$ cholesterol, $8 \%$ lard $(\mathrm{w} / \mathrm{w})$ and $0.05 \%$ cholate $(\mathrm{w} / \mathrm{w})$; simvastatin group animals (S; $n=10$ ) were fed with the same high fat diet plus simvastatin ( $2 \mathrm{mg} / \mathrm{kg} /$ day); and GBE group animals (GBE; $n=10$ ) were fed with the same high fat diet plus GBE (3 mg/kg/day). Each rabbit consumed about $120 \mathrm{~g}$ of food daily. Rabbits were caged individually with water ad libitum for 12 weeks, and maintained on a 12-h day/night cycle. Animal procedures were performed in accordance with guidelines set by the Animal Experiment Committee of Fudan University, Shanghai, China.

\section{Evaluation of atherosclerotic plaques}

To measure the atherosclerotic plaques, two complementary approaches were used: en face whole and histological section analyses [27]. The en face approach was employed to analyze the distribution and extent of atherosclerosis in the aorta, whereas microscopic analysis was used to evaluate lesion complexity as previously described [28]. In brief, the entire aorta was opened longitudinally and stained with Sudan IV-Herxheimer's solution (Sigma). Lesion area was determined by calculating the proportion of aortic intimal surface area occupied by the red stain in the descending thoracic aorta, and abdominal aorta with the use of a video-based image analysis program (MCID, Imaging Research, St Catharines, Ontario, Canada).

Next, all aortic segments were embedded in paraffin, and 4- $\mu \mathrm{m}$ thick cross-sectional serial sections were obtained [29]. To quantify luminal crosssectional area involved by atherosclerotic plaque, 10 sections obtained every $20 \mu \mathrm{m}$ from the arteries were stained with hematoxylin and eosin. Lesion areas were quantitated by light microscopy (Olympus BX40). Lesion surface area and total aortic crosssectional area were measured using Image Pro Plus version 6 (Media Cybernetics, Bethesda, MD).

\section{Plasma lipid analysis}

Plasma collected from 8 rabbits from each group was used for analysis of lipid compositions. Plasma total cholesterol (TC), triglyceride (TG), and low-den- 
sity lipoprotein cholesterol (LDL-C) were analyzed by enzyme-linked immunosorbent assay (ELISA) [30]. The concentrations were subsequently determined using a spectrophotometer (Roche/Hitachi analyzer, Roche Diagnostics, Indianapolis, IN, USA).

\section{Immunohistochemistry}

Four sections from eight rabbits per group were analyzed by immunohistochemical staining. Paraffin-embedded artery sections were deparaffinized by immersion in xylene, followed by a series of alcohol treatments. Endogenous peroxidase activity was quenched by immersing the slides in $0.3 \%$ hydrogen peroxide in methanol for $15 \mathrm{~min}$. The sections were rinsed in PBS three times for 5 min each and were blocked with $5 \%$ normal goat serum for 30 min. Primary antibodies were incubated for $90 \mathrm{~min}$ (goat anti-rabbit connexin 43 (Cx43), USCNLIFE, China, $1: 200$ dilution). Sections were washed and incubated with HRP-conjugated secondary antibodies for $30 \mathrm{~min}$. Staining was detected using the Alkaline Phosphatase Standard ABC Kit (Vector Labs, Burlingame, CA). The slides were counterstained with hematoxylin (Sigma, St. Louis, MO, USA). For quantification, pictures were captured and pixels were counted using Image Pro Plus Version 6.0 software (MediaCybernetics Inc., Bethesda, MD).

\section{Western blot analysis}

Total protein was isolated from arteries, and equal amounts $(20 \mu \mathrm{g})$ of protein samples were resolved by sodium dodecyl sulfate polyacrylamide gel electrophoresis (SDS-PAGE) and transferred onto a nitrocellulose membrane. The membrane was blocked for $1 \mathrm{~h}$ in 5\% milk in TBS-T (Tris buffered saline and 0.1\% Tween 20) and then incubated with primary antibody against Cx43 (1: 200; USCNLIFE) or $\beta$-actin ( 1 : 200; USCNLIFE) as an internal control overnight at $4^{\circ} \mathrm{C}$, followed by incubation with horseradish peroxidase-conjugated secondary antibody (1: 10,000; USCNLIFE). After washing 3 times with TBS, the membrane was developed using an enhanced chemiluminescent detection system (Amersham, Piscataway, NJ, USA). Signal intensities were quantitated using the Quantity One software (Bio-Rad, Hercules, CA).

\section{Statistical analysis}

All statistical analyses were carried out using the SPSS 11 software. If not otherwise stated, means of at least three independent experiments \pm SD are shown. Statistical evaluations were done using analysis of variance (ANOVA) with the Tukey post hoc test. Values of $p$ less than 0.05 were considered as significant.

\section{Results}

\section{Effects of GBE on serum lipid composition in rabbit}

Before treatment, the baseline serum lipid levels (TG, TC, and LDL-C) were comparable among all experimental groups and there were no obvious changes of serum lipid levels (TG, TC, and LDL-C) in the control group after 6 and 12 weeks (data not shown). However, after the indicated treatment for 6 weeks, GBE lowered high-fat diet-induced increase of serum TC by $24.4 \%$ (32.3 \pm 1.6 vs. 42.7 $\pm 2.6 \mathrm{mmol} / \mathrm{l} ; p<0.01$; Table I). Moreover, GBE decreased high-fat diet-induced increase of serum TG, $\mathrm{TC}$, and LDL-C by $59.1 \%$ (0.9 $\pm 0.2 \mathrm{mmol} / \mathrm{l}$ vs. 2.2 $\pm 0.4 \mathrm{mmol} / \mathrm{l}), 18.2 \%(31.1 \pm 1.4 \mathrm{mmol} / \mathrm{l}$ vs. 38.0 $\pm 0.4 \mathrm{mmol} / \mathrm{l})$ and $15 \%(28.9 \pm 1.3 \mathrm{mmol} / \mathrm{l}$ vs. 34.0 $\pm 1.0 \mathrm{mmol} / \mathrm{l})$, respectively, at 12 weeks $(p<0.01$; Table I).

\section{Effects of GBE on development of aortic atherosclerotic lesions with high-fat diet in rabbits}

To examine the effect of GBE on atherosclerotic lesion development in rabbits, we used GBE to treat the rabbits (GBE group) with a high-fat diet. Simvastatin-treated rabbits (S group) were used as a positive control. The en face Sudan IV-positive lesion areas of the aorta in the rabbits with a highfat diet (HF group, $88.2 \pm 2.2 \%$ ) were significantly larger than those in the rabbits with a normal-fat diet (control group, $\mathrm{C}$; 0$)$ ( $p<0.01$; Figure 1$)$, indicating that HF-induced atherosclerosis was successfully set up. The lesion areas in both S (40.8 $\pm 2.4 \%)$ and GBE $(51.7 \pm 3.1 \%)$ groups were significantly decreased relative to those in the HF group $(88.2 \pm 2.2 \% ; p<0.01$; Figure 1). Atherosclerotic

Table I. Effect of Ginkgo biloba extract on serum lipid composition changes in rabbits (mmol/l)

\begin{tabular}{|c|c|c|c|c|c|c|}
\hline \multirow[t]{2}{*}{ Parameter } & \multicolumn{3}{|c|}{6 weeks } & \multicolumn{3}{|c|}{12 weeks } \\
\hline & HF & $S$ & GBE & HF & $\mathrm{S}$ & GBE \\
\hline TG & $0.8 \pm 0.2$ & No change* & $0.9 \pm 0.1$ & $2.2 \pm 0.4$ & No change* & $0.9 \pm 0.2^{*}$ \\
\hline $\mathrm{TC}$ & $42.7 \pm 2.6$ & $22.3 \pm 1.4^{\star}$ & $32.3 \pm 1.6^{*}$ & $38.0 \pm 0.4$ & $20.2 \pm 2.0^{\star}$ & $31.1 \pm 1.4^{*}$ \\
\hline LDL-C & $28.1 \pm 1.8$ & $18.4 \pm 0.5^{\star}$ & $27.4 \pm 0.2$ & $34.0 \pm 1.0$ & $15.7 \pm 1.6^{\star}$ & $28.9 \pm 1.3^{*}$ \\
\hline
\end{tabular}



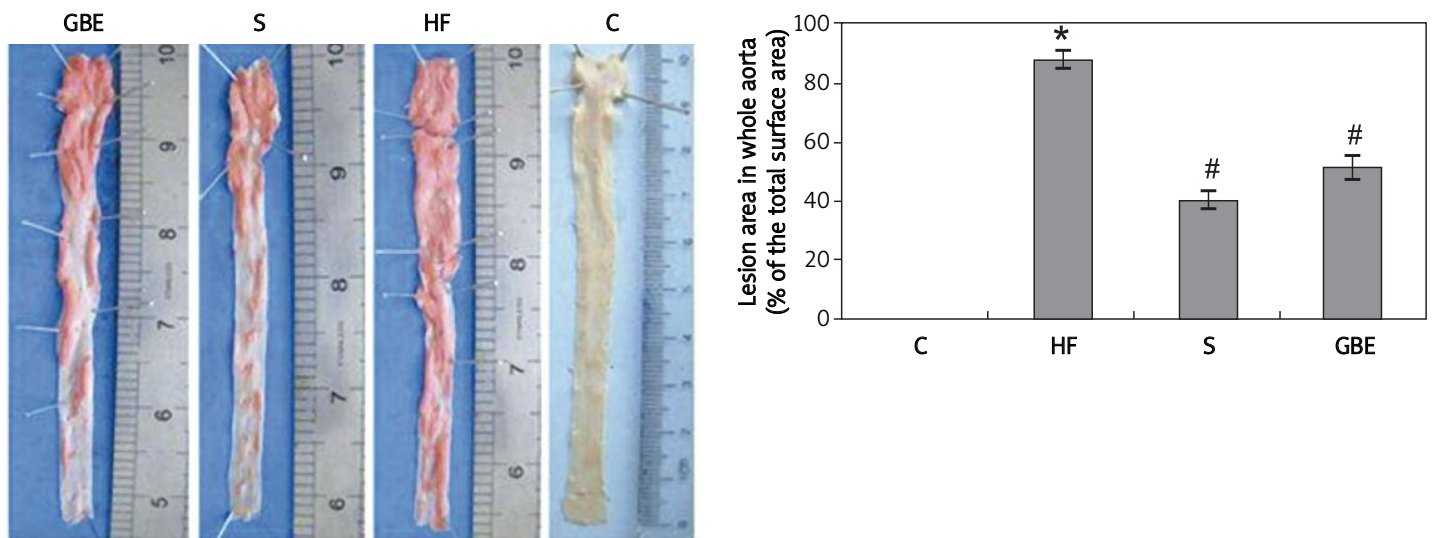

Figure 1. Effects of GBE on plaque formation in rabbits. Representative examples of en face dissection of thoracic and abdominal aorta, stained with Sudan IV, showed atherosclerotic lesions (red). Atherosclerotic involvement for each rabbit was quantitatively determined in en face sections by measuring the proportion of aortic intimal surface area occupied by Sudan IV positive lesions in total aorta

$C$ - normal diet control group, HF - high-fat diet group, S - simvastatin treated group, GBE - Ginkgo biloba extract treated group; results were expressed as mean $\pm S D ;{ }^{*} p<0.01$ relative to normal diet control (C), ${ }^{*} p<0.01$ relative to high-fat diet group (HF)
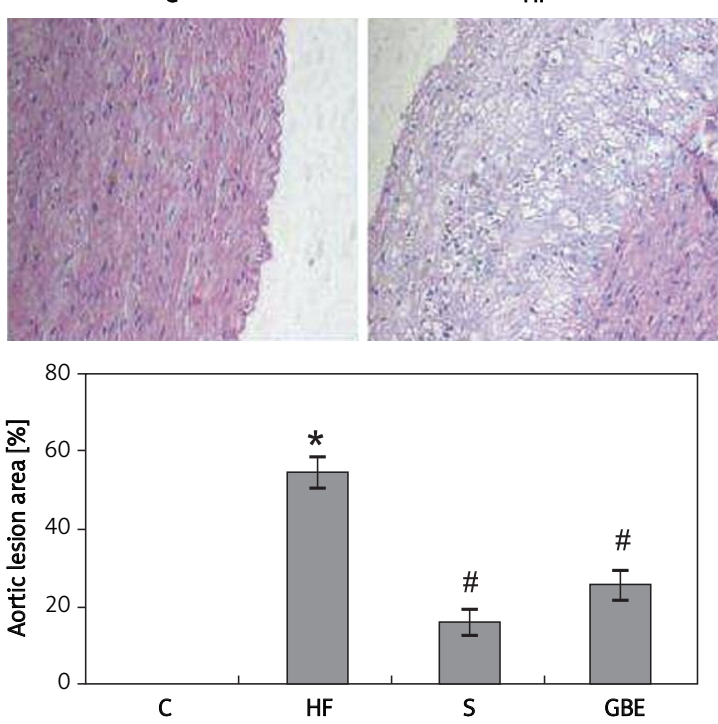

lesion development in the aorta was also analyzed by $\mathrm{H}+\mathrm{E}$ staining (Figure 2). Mean atherosclerotic lesion area in the GBE group was reduced by $53.2 \%$ compared with the HF group ( $25.7 \pm 3.8 \%$ vs. 54.9 $\pm 4.0 \% ; p<0.01$; Figure 2).

\section{GBE treatment reduced the expression of Cx43}

Gap junction protein Cx43 plays a key role in atherogenesis [31-34]. To explore the mechanism of GBE-induced suppression of atherosclerotic development, we analyzed the effect of GBE on the expression of $\mathrm{C} \times 43$ by immunohistochemistry in the rabbits with a high-fat diet. Figure 3 shows that Cx43 expression in the HF group was significantly augmented. GBE significantly diminished high-fat diet-induced Cx43 expression. Western blot analysis was also performed to measure the expression of $\mathrm{Cx} 43$ (Figure 4). In line with immunohistochem- $\mathrm{s}$
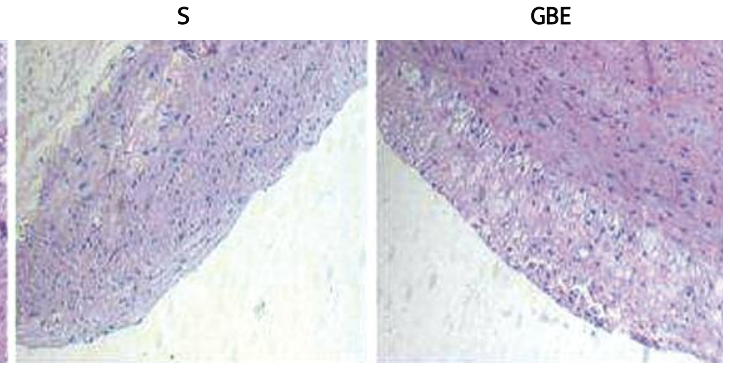

Figure 2. GBE inhibits atherosclerotic lesion development in the aorta of rabbits with high-fat diet. Representative hematoxylin and eosin stained histological cross sections from aorta of indicated groups

Abbreviations - see Figure 1. Results were expressed as mean $\pm S D ;{ }^{*} p<0.01$ relative to normal diet control $(C),{ }^{\#} p<0.01$ relative to high-fat diet group (HF). Magnification 100x

istry results, high-fat diet-induced Cx43 expression level was also downregulated in GBE groups.

\section{Discussion}

Ginkgo biloba extract EGb761 has antioxidant and antiplatelet aggregation effects indicating that it might protect against atherosclerosis [35]. Our results documented that GBE significantly decreased TC, LDL-C and TG levels, and attenuated atherosclerotic lesion development in the aorta, indicating potential anti-hyperlipidemic and antiatherosclerotic effects of GBE. Although it seems that the anti-hyperlipidemic and anti-atherosclerotic properties of GBE are not as efficiency as simvastatin, GBE is extracted from Ginkgo biloba leaves with less side effects and has been widely used as a therapeutic agent for cardiovascular disorders [16]. Moreover, GBE has been suggested to be used in 
C

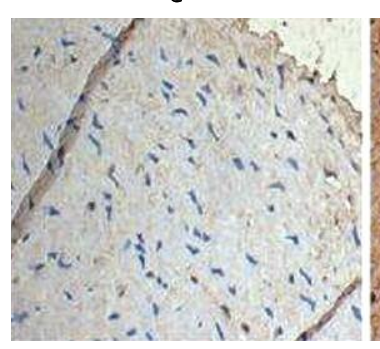

HF
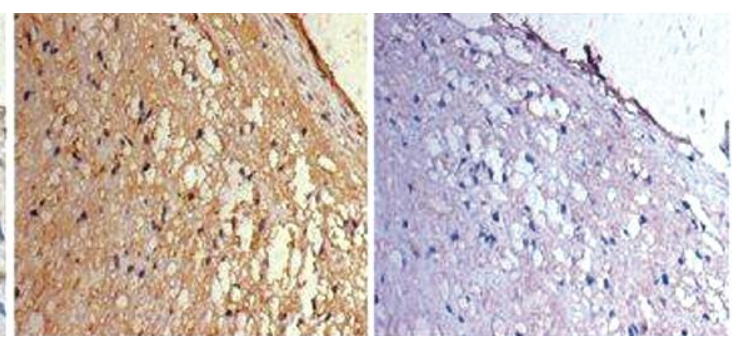

GBE

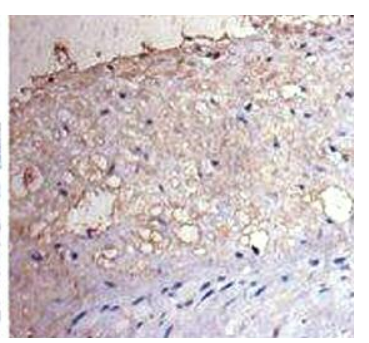

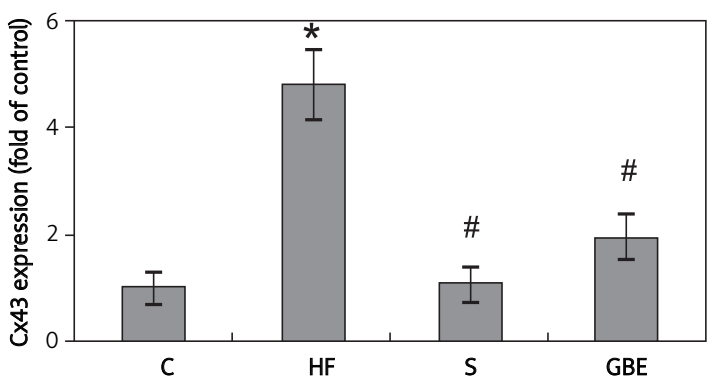

Figure 3. Expression of connexin $43(\mathrm{C} \times 43)$ in atherosclerotic plaques in rabbits. Representative sections show immunohistochemistry for $\mathrm{Cx} 43$ (left). Sections from thoracic and abdominal aorta were stained for $\mathrm{Cx} 43$. Positive staining is shown as brown. Sections were counterstained with hematoxylin. Quantitative analysis (fold of normal diet control group) of $\mathrm{Cx} 43$ expression in different experimental groups was performed (right). The expression of Cx43 was significantly increased in the rabbits with a high-fat diet compared to that in the rabbits with a normal diet. The expression of $\mathrm{Cx} 43$ in both simvastatin and Ginkgo biloba extract groups was decreased relative to that of the high-fat diet control group

The data are expressed in terms of the fold of the normal diet control group as mean $\pm S D$; ${ }^{*} p<0.01$ relative to normal diet control (C), ${ }^{*} p<0.01$ relative to high-fat diet group (HF). Magnification $200 x$
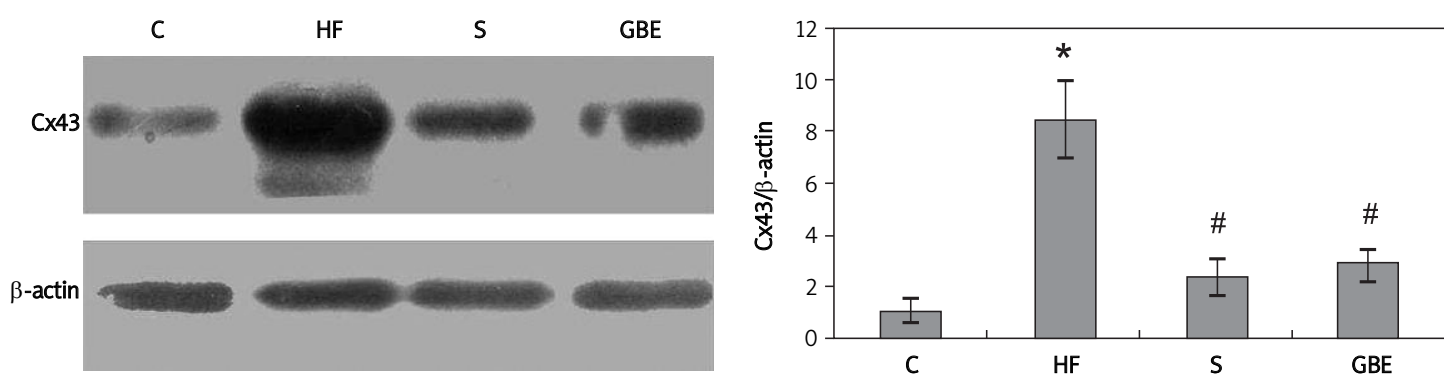

Figure 4. Western blot analysis of connexin 43 (Cx43) expression. Top panel shows a representative blot of Cx43 protein. $\beta$-actin was used as an internal control. The normalized $C \times 43$ protein levels are shown in the bottom panel Abbreviations - see Figure 1. Results were expressed as mean $\pm S D$; ${ }^{*} p<0.01$ relative to normal diet control $(C),{ }^{*} p<0.01$ relative to high-fat diet group (HF)

arteriosclerosis prophylaxis in patients [18]. All of the studies indicate GBE as a promising anti-atherosclerotic drug.

It has been suggested that a distinctive spatial and temporal pattern of $\mathrm{Cx} 43$ expression between smooth muscle cells accompanies development of the atherosclerotic plaque [36]. Moreover, reduced Cx43 expression inhibits atherosclerotic lesion formation in low-density lipoprotein receptor-deficient mice [37]. Thus, $C \times 43$ is a cardiovascular risk marker and therapeutic target [38]. Statins have been suggested as potent connexin modulators, synergizing with its lipid-lowering effect to reduce atherosclerosis mortality [39]. To investigate the mechanism of GBE-mediated suppression of atherosclerotic progress, we measured the expression of Cx43. We found that GBE treatment suppressed
Cx43 expression, indicating another mechanism of GBE-mediated inhibition of atherosclerosis. In contrast to the suppressive effect of GBE on the expression of $\mathrm{Cx} 43$ in atherosclerosis, GBE could exert its neuroprotective effects by improvement of $\mathrm{C} \times 43$ expression and gap junction intercellular communication (GIIC) induced by hypoxia/ischemia-reoxygenation/reperfusion injury [40]. The different effects of GBE on the expression of $\mathrm{C} \times 43$ indicate that $G B E$ regulates $C \times 43$ through multiple pathways.

Although we found that GBE significantly inhibits high-fat diet-induced atherosclerosis via suppressing the expression of $\mathrm{Cx} 43$, there are still some open questions. One would be how GBE inhibits Cx43 expression in animal models of high-fat dietinduced atherosclerosis. Another one is whether the downregulation of $\mathrm{Cx} 43$ is sufficient for GBE- 
mediated protective effects against high-fat dietinduced atherosclerosis. More attention will be focused on the molecular mechanisms of how GBE prevents the development of atherosclerotic plaques in the near future.

In conclusion, our study documents that GBE prevents atherosclerosis progress through modulating plasma lipid, suppressing atherosclerotic lesion development and Cx43 expression.

\section{Acknowledgments}

Jian Ming Wei and Xin Wang contributed equally to this work.

This work was supported in part by grants from Shanghai Municipal Health Bureau (2009036) and from Jinshan District Health Bureau of Shanghai (200923) to Jian Ming Wei.

\section{References}

1. Lewis DR, Kamisoglu K, York AW, Moghe PV. Polymerbased therapeutics: nanoassemblies and nanoparticles for management of atherosclerosis. Wiley Interdiscip Rev Nanomed Nanobiotechnol 2011; 3: 400-20.

2. Rizzo M, Battista Rini G. Ezetimibe, cardiovascular risk and atherogenic dyslipidaemia. Arch Med Sci 2011; 7: 5-7.

3. Bucher HC, Griffith LE, Guyatt GH. Systematic review on the risk and benefit of different colesterol-lowering interventions. Arterioscler Thromb Vasc Biol 1999; 19: 187-95.

4. Gotto AM. Antioxidants, statins, and atherosclerosis. J Am Coll Cardiol 2003; 41: 1205-10.

5. Kowalski J, Barylski M, Banach M, Grycewicz J, Irzmański R, Pawlicki L. Neutrophil superoxide anion generation during atorvastatin and fluvastatin therapy used in coronary heart disease primary prevention. J Cardiovasc Pharmacol 2006; 48: 143-7.

6. Aydin U, Ugurlucan M, Gungor F, et al. Effects of atorvastatin on vascular intimal hyperplasia: an experimental rodent model. Angiology 2009; 60: 370-7.

7. Mieczkowska J, Mosiewicz J, Barud W, Kwaśniewski W. Changes in the activity of connective tissue matrix enzymes in the metabolic syndrome. Arch Med Sci 2011; 7: 634-41.

8. Szulc M, Radwan-Oczko M. The role of Porphyromonas gingivalis in atherosclerosis. Kardiochir Torakochi 2012; 9: 100-5.

9. Klingenberg R, Hansson GK. Treating inflammation in atherosclerotic cardiovascular disease:emerging therapies. Eur Heart J 2009; 30: 2838-44.

10. Bielecka-Dabrowa A, Barylski M, Mikhailidis DP, Rysz J, Banach M. HSP 70 and atherosclerosis: protector or activator? Expert Opin Ther Targets 2009; 13: 307-17.

11. Sheikine YA, Hansson GK. Chemokines as potential therapeutic targets in atherosclerosis. Current Drug Targets 2006; 7: 13-28.

12. Krotz F, Sohn HY, Klauss V. Antiplatelet drugs in cardiological practice: established strategies and new developments. Vasc Health Risk Manag 2008; 4: 637-45.

13. Gonzalez ER. Antiplatelet therapy in atherosclerotic cardiovascular disease. Clin Ther 1998; 20: B18-41.

14. Jawień J, Olszanecki R, Nowak B, Marcinkiewicz J. Apolipoprotein E knockout mice: an experimental model to study inflammatory mechanisms of atherosclerosis and to screen a putative anti-atherogenic properties of drugs. Centr Eur J Immunol 2012; 37: 36-44.

15. Otocka-Kmiecik A, Mikhailidis DP, Nicholls SJ, Davidson M, Rysz J, Banach M. Dysfunctional HDL: a novel important diagnostic and therapeutic target in cardiovascular disease? Prog Lipid Res 2012; 51: 314-24.

16. Ou HC, Lee WJ, Lee IT, et al. Ginkgo biloba extract attenuates oxLDL-induced oxidative functional damages in endothelial cells. J Appl Physiol 2009; 106: 1674-85.

17. Rodríguez M, Ringstad L, Schäfer $P$, et al. Reduction of atherosclerotic nanoplaque formation and size by Ginkgo biloba (EGb 761) in cardiovascular high-risk patients. Atherosclerosis 2007; 192: 438-44.

18. Siegel G, Schäfer P, Winkler K, Malmsten M. Ginkgo biloba (EGb 761) in arteriosclerosis prophylaxis. Wien Med Wochenschr 2007; 157: 288-94.

19. Wei Z, Peng Q, Lau BH, Shah V. Ginkgo biloba inhibits hydrogen peroxide-induced activation of nuclear factor kappa B in vascular endothelial cells. Gen Pharmacol 1999; 33: 369-75.

20. Chen JW, Chen YH, Lin FY, Chen YL, Lin SJ. Ginkgo biloba extract inhibits tumor necrosis factor-alpha-induced reactive oxygen species generation, transcription factor activation, and cell adhesion molecule expression in human aortic endothelial cells. Arterioscler Thromb Vasc Biol 2003; 23: 1559-66.

21. Jiao YB, Rui YC, Li TJ, Yang PY, Qiu Y. Expression of proinflammatory and anti-inflammatory cytokines in brain of atherosclerotic rats and effects of Ginkgo biloba extract. Acta Pharmacol Sin 2005; 26: 835-9.

22. Pierre SV, Lesnik P, Moreau M, et al. The standardized Ginkgo biloba extract Egb-761 protects vascular endothelium exposed to oxidized low density lipoproteins. Cell Mol Biol (Noisy-le-grand) 2008; 54 Suppl: OL1032-42.

23. Liu F, Zhang J, Yu S, et al. Inhibitory effect of Ginkgo biloba extract on hyperhomocysteinemia-induced intimal thickening in rabbit abdominal aorta after balloon injury. Phytother Res 2008; 22: 506-10.

24. Liu HJ, Wang XL, Zhang L, et al. Inhibitions of vascular endothelial growth factor expression and foam cell formation by EGb 761, a special extract of Ginkgo biloba, in oxidatively modified low-density lipoprotein-induced human THP-1 monocytes cells. Phytomedicine 2009; 16: 138-45.

25. Qiu Y, Rui YC, Li TJ, Zhang L, Yao PY. Inhibitory effect of extracts of Ginkgo biloba leaves on VEGF-induced hyperpermeability of bovine coronary endothelial cells in vitro. Acta Pharmacol Sin 2004; 25: 1306-11.

26. Tsai JY, Su KH, Shyue SK, et al. EGb761 ameliorates the formation of foam cells by regulating the expression of SR-A and ABCA1: role of haem oxygenase-1. Cardiovasc Res 2010; 88: 415-23.

27. Secchiero P, Candido R, Corallini F, et al. Systemic tumor necrosis factor-related apoptosis-inducing ligand delivery shows antiatherosclerotic activity in apolipoprotein E-null diabetic mice. Circulation 2006; 114: 1522-30.

28. Candido R, Allen TJ, Lassila M, et al. Irbesartan but not amlodipine suppresses diabetes associated atherosclerosis. Circulation 2004; 109: 1536-42.

29. Glover LE, Newton K, Krishnan G, et al. Dysferlin overexpression in skeletal muscle produces a progressive myopathy. Ann Neurol 2010; 67: 384-93.

30. Davis MC, Zautra AJ, Younger J, Motivala SJ, Attrep J, Irwin MR. Chronic stress and regulation of cellular markers of inflammation in rheumatoid arthritis: implications for fatigue. Brain Behav Immun 2008; 22: 24-32. 
31. Wang LH, Chen JZ, Sun YL, et al. Statins reduce connexin 40 and connexin43 expression in atherosclerotic aorta of rabbits. Int J Cardiol 2005; 100: 467-75.

32. Kwak BR, Veillard N, Pelli G, et al. Reduced connexin43 expression inhibits atherosclerotic lesion formation in low-density lipoprotein receptor-deficient mice. Circulation 2003; 107: 1033-9.

33. Polacek D, Bech F, McKinsey JF, Davies PF. Connexin43 gene expression in the rabbit arterial wall: effects of hypercholesterolemia, balloon injury and their combination. J Vasc Res 1997; 34: 19-30.

34. Blackburn JP, Peters NS, Yeh HI, Rothery S, Green CR, Severs NJ. Upregulation of connexin43 gap junctions during early stages of human coronary atherosclerosis. Arterioscler Thromb Vasc Biol 1995; 15: 1219-28.

35. Lim S, Yoon JW, Kang SM, et al. EGb761, a Ginkgo biloba extract, is effective against atherosclerosis in vitro, and in a rat model of type 2 diabetes. PLoS One 2011; 6 : e20301.

36. Blackburn JP, Peters NS, Yeh HI, Rothery S, Green CR, Severs NJ. Upregulation of connexin43 gap junctions during early stages of human coronary atherosclerosis. Arterioscler Thromb Vasc Biol 1995; 15: 1219-28.

37. Kwak BR, Veillard N, Pelli G, et al. Reduced connexin43 expression inhibits atherosclerotic lesion formation in low-density lipoprotein receptor-deficient mice. Circulation 2003; 107: 1033-9.

38. Burnier L, Fontana P, Angelillo-Scherrer A, Kwak BR. Intercellular communication in atherosclerosis. Physiology (Bethesda) 2009; 24: 36-44.

39. Kwak BR, Veillard N, Pelli G, et al. Reduced connexin 43 expression inhibits atherosclerotic lesion formation in low-density lipoprotein receptor-deficient mice. Circulation 2003; 107: 1033-9.

40. Li Z, Lin XM, Gong PL, Zeng FD, Du GH. Effects of Gingko biloba extract on gap junction changes induced by reperfusion/reoxygenation after ischemia/hypoxia in rat brain. Am J Chin Med 2005; 33: 923-34. 